POS PROCEEDINGS

\title{
Mapping the densities of exotic nuclei
}

\section{S. Karataglidis*}

Department of Physics, University of Johannesburg, P.O. Box 524, Auckland Park, 2006, South

Africa, and

School of Physics, University of Melbourne, Victoria, 3010, Australia

E-mail: stevenkaduj.ac.za

\begin{abstract}
Measurements of electron scattering form factors from exotic nuclei are becoming a reality with the development of the SCRIT Project at RIKEN, as well as the possibility of the ELISe project at FAIR/GSI. Such measurements will elicit directly information of the charge densities of exotic nuclei. In the case of neutron-rich exotic nuclei, most of the studies have concentrated on the skin or halo aspects of the neutron density, while treating the proton density as contained in the core. Very little information has been obtained about the proton density in exotic neutron-rich nuclei, as a result. Conversely, intermediate energy proton scattering in the inverse kinematics, with typical energies of around $40 \mathrm{~A} \mathrm{MeV}$, provides some information on the proton density, but as the effective nucleon-nucleon interaction at intermediate energies is dominated by the protonneutron interaction, that scattering also gives information primarily of the neutron density. Results of calculations of elastic and inelastic electron scattering form factors for the exotic isotopes of $\mathrm{He}$ and $\mathrm{Li}$ will be presented and discussed, as well as results for other light exotic nuclei, in order to highlight the information one may obtain from the new facilities. Together with relevant intermediate energy proton scattering data, one may finally have a complete picture of the matter densities of exotic nuclei. The impact this will have on the descriptions of the structures of exotic nuclei will be discussed.
\end{abstract}

The 26th International Nuclear Physics Conference

11-16 September, 2016

Adelaide, Australia

\footnotetext{
*Speaker.

${ }^{\dagger}$ Support from the National Research Foundation of South Africa is acknowledged.
} 


\section{Introduction}

Much information has been obtained regarding the (one-body) neutron densities of (neutronrich) exotic nuclei, highlighting the emergent phenomena of halos and skins. In large part, such information has been obtained from peripheral collisions in heavy-ion scattering, which have only been able to obtain information on the asymptotic features of the neutron density. Light exotic nucleus scattering from hydrogen at intermediate energies, which in the inverse kinematics transforms to proton scattering from the nucleus, gives information also of the neutron density in the core, as the effective nucleon-nucleon interaction is dominated by the proton-neutron component. In order to evaluate models of structure for exotic nuclei, particularly at the level of nucleonic degrees of freedom, detailed information of both the proton and neutron densities are required.

Yet there is very little information available for the proton densities of exotic nuclei. This is largely due to the problem of the creation of proton-rich exotic nuclei in the laboratory. The possibility exists for $(p, n)$ or $(n, p)$ charge exchange reactions, or the use of $\left(\gamma, \pi^{ \pm}\right)$reactions (see, for example, Refs. [四] and [వ]), which can obtain information on the structure of exotic final states, particularly in stable target experiments. There is now the possibility of directly measuring the charge densities of exotic nuclei, which relate to the proton densities, by means of electron scattering, with the SCRIT facility at RIKEN [B]] in operation, and the FAIR/ELISe [四] in proposal. The SCRIT facility will concentrate on the measurements of the charge form factors for mediummass exotic nuclei, which are produced by the photofission of uranium, while the ELISe facility will be able to access light exotic nuclei. Some theoretical predictions have been made recently in order to understand what information such experiments may yield, and it is opportune to review those predictions. Together with the knowledge of the neutron densities, one may then be able to map the one-body matter densities of exotic nuclei which will provide sensitive tests of models of structure used in the specifications of such nuclei.

In order for such a mapping to be possible, however, one must start with a credible, nucleonbased, model of structure, by which then predictions of both intermediate-energy proton and electron scattering may be obtained self-consistently. It is this aspect which necessarily requires the use of microscopic models of the structure of exotic nuclei. As analyses of proton scattering data from exotic nuclei have been analysed microscopically (see, for example, Refs. [可] and [回], which firmly established ${ }^{6} \mathrm{He}$ as a halo nucleus), herein I will concentrate on the specifics of predictions of electron scattering form factors, referring to relevant proton scattering data where necessary.

\section{Proton and electron scattering}

As stated above, a self-consistent description of both proton and electron scattering requires a microscopic (nucleon-based) model of structure for the specification of the target. While SkyrmeHartree-Fock models have been used for heavy mass nuclei [ [ $]$ ], the results presented herein assume the shell model for the base model of structure for the exotic nuclei. A microscopic, nonrelativistic, approach for the (parameter-free) specification of the intermediate-energy nucleon-nucleus optical potential has been used for the analyses of proton scattering data [ $[8]$. The specification of the optical potential requires the use of the one-body (transition) density matrix elements (OBDME), 
defined by

$$
S_{j_{1} j_{2} J}=\left\langle J_{f}\left\|\left[a_{j_{1}}^{\dagger} \times \tilde{a}_{j_{2}}\right]^{J}\right\| J_{i}\right\rangle,
$$

for a single particle transition between shell model single-particle states $j_{2}$ and $j_{1}$, defining a transition between nuclear states $J_{i}$ and $J_{f}$. These can also be defined for elastic scattering accordingly.

Longitudinal and transverse electron scattering form factors have been obtained using the microscopic model of deForest and Walecka [Q], as extended by Karataglidis, Halse, and Amos [ए]] to include implicitly meson exchange currents in the transverse electric form factors using Siegert's theorem. Central to the use of this model, which calculates the matrix elements for the scattering directly without recourse to calculating a Fourier/Bessel transform of the radial one-body charge density, is the use of the same OBDME as used in the calculations of the observables in proton scattering, providing a direct connection between electron and proton scattering.

Analyses of the complementary electron and proton scattering data may thus be done using the same underlying model of structure, from which one may obtain a map of the matter density for the nucleus in question.

\section{Results from other groups}

Given that there have not been any electron scattering data for exotic nuclei forthcoming from experimental facilities at this stage, the study of the form factors of exotic nuclei have been limited to theoretical study. There have been some studies of the form factors of intermediate energy form factors for medium- and heavy-mass nuclei (see for example Refs [ए], ए2, [3]]) and for the so-called "bubble" nuclei [[13]. However, very little attention has been given to the light nuclei. Studies of the neutron-rich $\mathrm{He}$ and $\mathrm{Li}$ isotopes, as well as the proton-rich halo nucleus ${ }^{8} \mathrm{~B}$, has been done by [122, [3], ㄴ5].

Antonov et al. [ए2] predicted the charge form factors for ${ }^{6,8} \mathrm{He}$ and ${ }^{8,9,11} \mathrm{Li}$ by obtaining the charge densities of those systems using the large-space shell model wave functions of Karataglidis et al. [16, [ [, [1]]. The form factors are then found by a Fourier transform of the (point) densities of the protons and neutrons, correcting for centre-of-mass and finite-size effects, using the Sachs proton and neutron electric form factors. They found a slightly smaller form factor for ${ }^{8} \mathrm{He}$ than for ${ }^{6} \mathrm{He}$, but they did not consider the possible effect of the extensive neutron density in ${ }^{6} \mathrm{He}$. A similar situation was found for the $\mathrm{Li}$ isotopes, whereupon the form factor for ${ }^{11} \mathrm{Li}$ was found to be smaller than that for ${ }^{6} \mathrm{Li}$. Again, the effect of the difference between an extensive neutron distribution (halo) and tight neutron distribution (skin) in the form factor of ${ }^{11} \mathrm{Li}$ was not investigated.

Bertulani [[13] found essentially similar results for the light nuclei, but found that the halo contribution to the form factor for ${ }^{11} \mathrm{Li}$ was essentially negligible. In the case of the form factor for ${ }^{8} \mathrm{~B}$, the effect of the proton halo is more pronounced, but still has little overall effect.

\section{Results}

(The results presented herein are taken from Ref. [[5]]. Work is currently underway to extend the formalism to medium mass nuclei, to analyse the form factors that may be obtained at the SCRIT Facility.) In order to examine the roles of both the halos and skins, one should begin with 
the form factor(s) of the stable isotope of the element, and then consider the addition of nucleons and their subsequent effect on the form factor. Such was done for the $\mathrm{He}$ and $\mathrm{Li}$ isotopes.

For the $\mathrm{He}$ case, we start with the form factor for ${ }^{4} \mathrm{He}$ and compare to those for ${ }^{6} \mathrm{He}$ and ${ }^{8} \mathrm{He}$. In all cases, the assumed structure was a $(0+2+4) \hbar \omega$ shell model using the Zheng interaction [ए8]]. Fig. $\square$ shows the experimental form factor for ${ }^{4} \mathrm{He}$ [एव], and compared to the shell-model

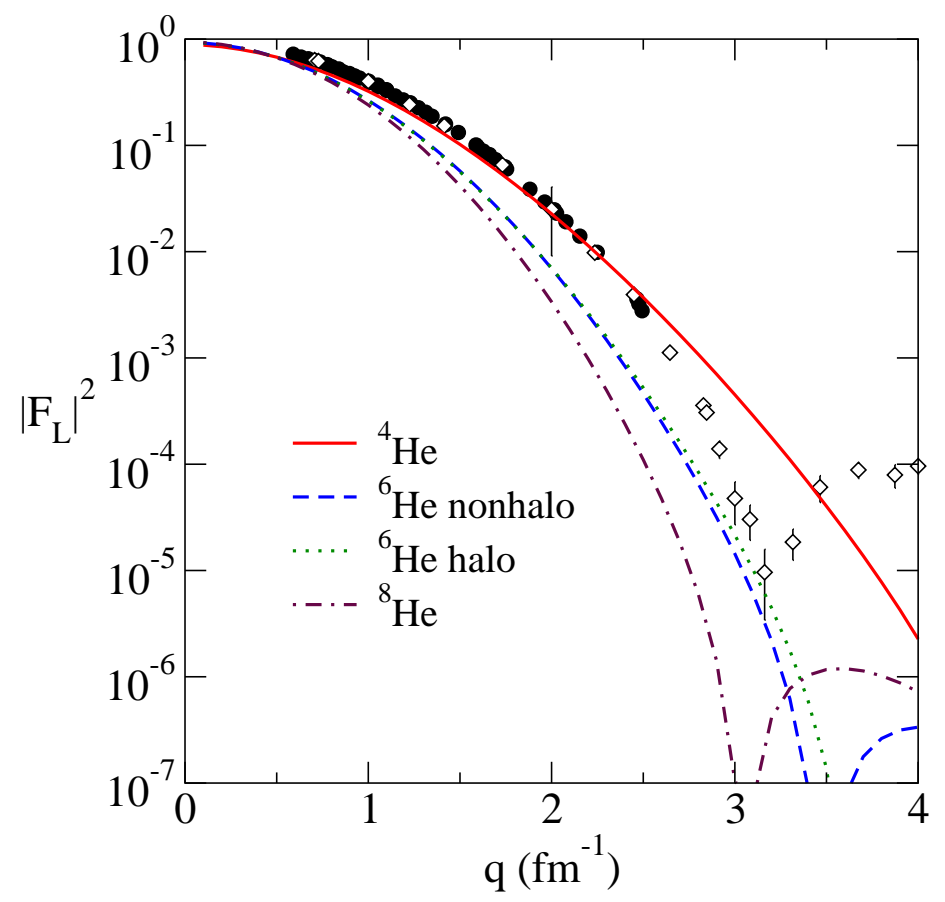

Figure 1: Elastic scattering form factors for ${ }^{4} \mathrm{He},{ }^{6} \mathrm{He}$, and ${ }^{8} \mathrm{He}$. The results obtained for ${ }^{4} \mathrm{He}$ and ${ }^{8} \mathrm{He}$ are given by the solid and dot-dashed lines, respectively. Two results are presented for that for ${ }^{6} \mathrm{He}$ : that obtained using Woods-Saxon single-particle wave functions (dotted line, "halo"), and that obtained using harmonic oscillator wave functions (dashed line, "nonhalo"). Details are given in the text.

result. Good agreement with the prediction is obtained up to $2.5 \mathrm{fm}^{-1}$; beyond this momentum transfer, meson-exchange currents need to be included [ए5]]. The form factors for ${ }^{6} \mathrm{He}$ and ${ }^{8} \mathrm{He}$ are concentrated more towards the smaller momentum transfers. There are two results shown for ${ }^{6} \mathrm{He}$ : the first is the "nonhalo" result, which uses the same harmonic oscillator wave functions as specified by the structure model, with an oscillator parameter of $1.8 \mathrm{fm}$, as was also used for the calculations of the form factors for ${ }^{4,8} \mathrm{He}$. The second result, specified by "halo", uses WoodsSaxon wave functions, where the binding energies of the orbits containing the valence neutrons are set to the single-neutron separation energy of $1.8 \mathrm{MeV}$. (This technique was used to explain the large $B(E 1)$ value in ${ }^{11} \mathrm{Be}[[\mathrm{ZO}]$, and has been used to analyse the elastic and inelastic intermediate energy proton scattering data from ${ }^{6} \mathrm{He}\left[\right.$ []].) The progression of the form factors from ${ }^{4} \mathrm{He}$ to ${ }^{8} \mathrm{He}$ is relatively smooth. Note, however, that the halo and nonhalo results for ${ }^{6} \mathrm{He}$ are very similar, indicating that the effect of the neutron halo is small. That is consistent with the conclusion of Bertulani.

The same procedure is followed for the Li isotopes, for which the elastic scattering (longitudi- 
nal and transverse) form factors were obtained for ${ }^{7} \mathrm{Li},{ }^{9} \mathrm{Li}$, and ${ }^{11} \mathrm{Li}$, where the binding energy for the valence neutrons in ${ }^{11} \mathrm{Li}$ has been set to the single-neutron separation energy of $396 \mathrm{keV}$. Fig. [ shows the longitudinal elastic scattering form factors for the three isotopes. The same conclusions

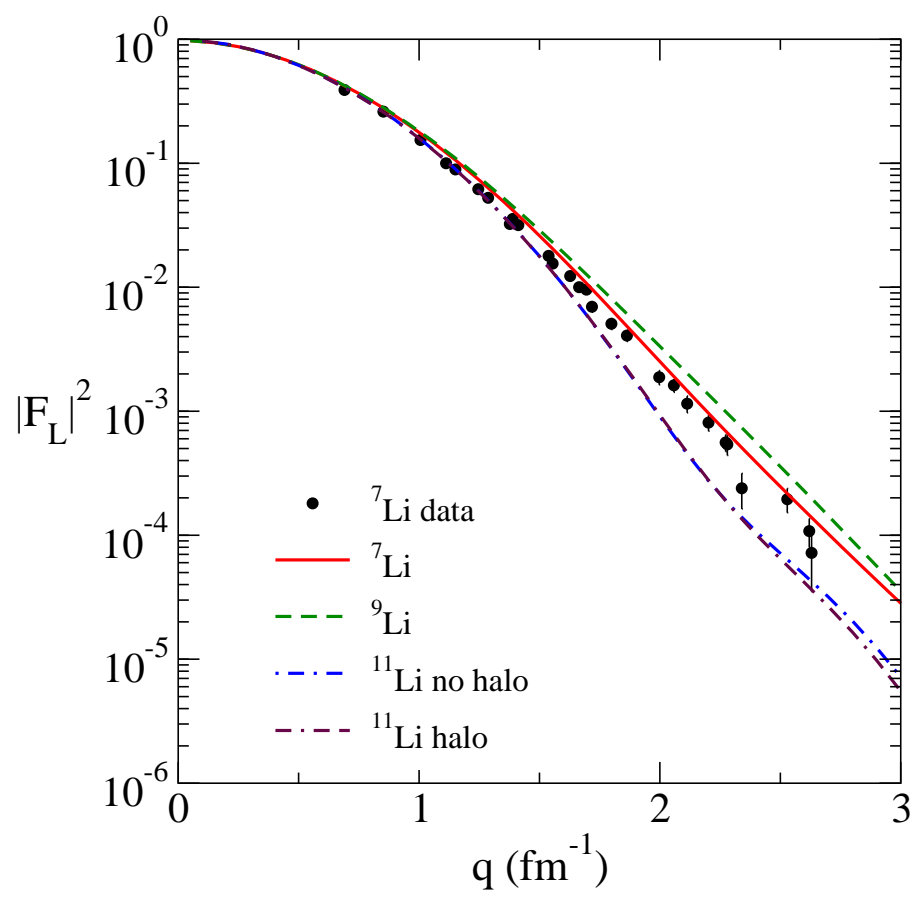

Figure 2: As for Fig. 凹, but for the longitudinal elastic scattering form factor for the Li isotopes. The data for the ${ }^{7} \mathrm{Li}$ form factor are from Refs. [[2], [22].

are reached as for the He isotopes, and the effect of the neutron halo in the longitudinal form factor for ${ }^{11} \mathrm{Li}$ is negligible. In the case of the transverse elastic scattering form factors, shown in Fig. B, all the transverse form factors are essentially equal. The neutron contribution to the $M 1$ transverse form factor for ${ }^{11} \mathrm{Li}$ is also shown at the bottom of the figure, and shows that the contribution of the neutrons to the transverse form factor is negligible.

By contrast, the elastic scattering form factors for ${ }^{8} \mathrm{~B}$ show a significant contribution from the halo proton. The longitudinal elastic scattering form factor for ${ }^{8} \mathrm{~B}$ is shown in Fig. 因. Clearly, the results show a significant effect of the proton halo in the longitudinal form factor for ${ }^{8} \mathrm{~B}$. The extension of the charge density results in a change in the charge form factor towards smaller momentum transfers, as one would expect by the Fourier transform. A significant change is also noticed in the transverse form factor [ㄷ]. Note that these effects are more pronounced than those observed by Bertulani [[3].

Comparing the results for the neutron-rich exotic nuclei with those for ${ }^{8} \mathrm{~B}$ shows that future experiments should concentrate on proton-rich exotic nuclei in measurements of form factors, especially if the nucleus is a proton halo. Effects of the neutron halo are negligible. Changes in the charge density along an isotonic chain would also be suggested, as the form factors should reflect the changes in the charge density. Where possible, these measurements should be complemented by equivalent intermediate energy proton scattering experiments, in order to assess the neutron 


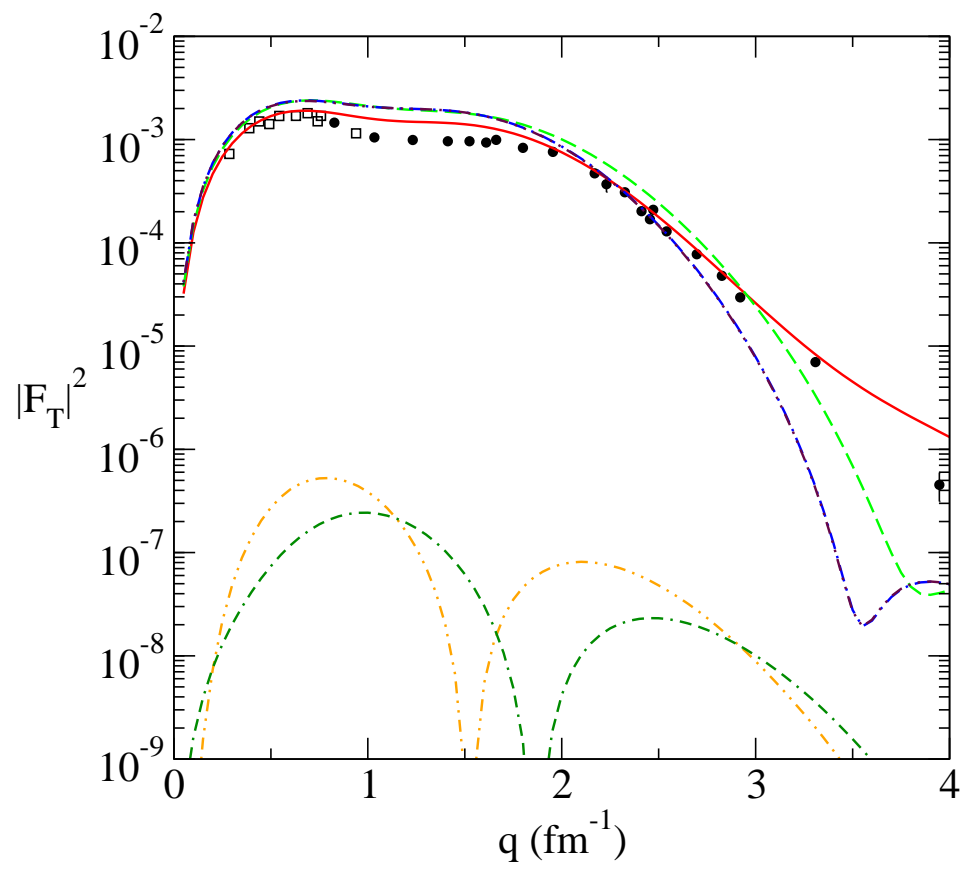

Figure 3: As for Fig. [, but for the transverse elastic scattering form factors. The data for the ${ }^{7} \mathrm{Li}$ form factor are from Refs. [[22, [23]].

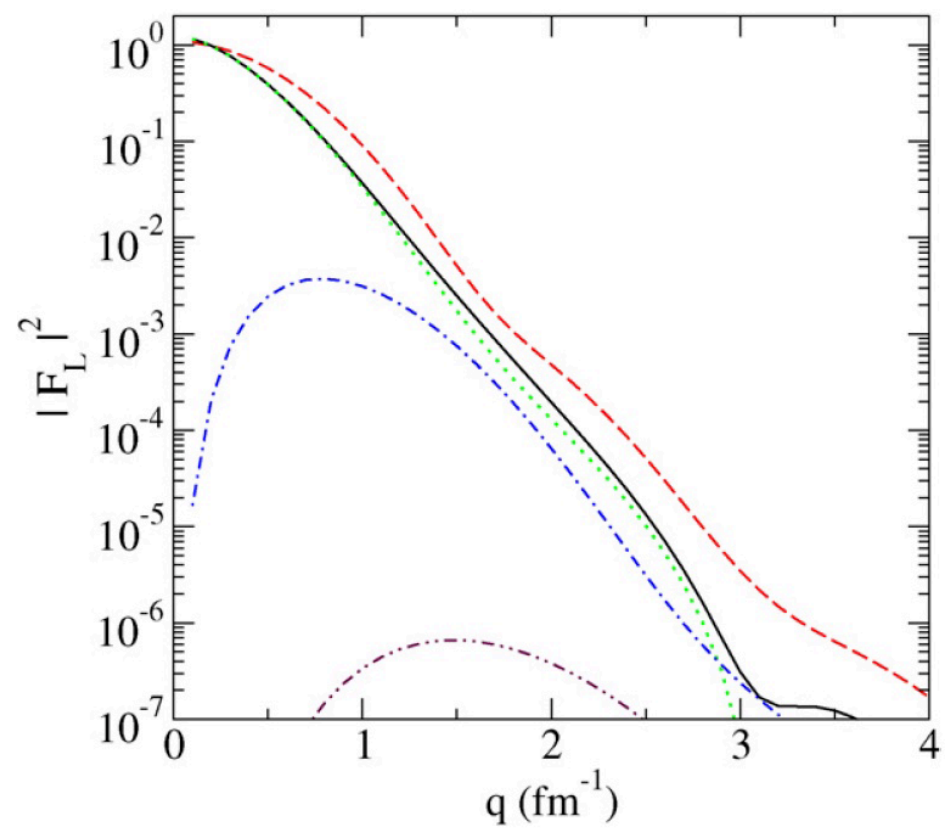

Figure 4: The longitudinal form factor for ${ }^{8} \mathrm{~B}$. The halo and nonhalo model results are given by the solid and dashed lined. The $C 0, C 2$, and $C 4$ components of the halo form factor are given by the dotted, dot-dashed, and double-dot-dashed lines, respectively. 
densities; together those results would provide a complete map of the matter densities of these systems.

\section{Conclusion}

Results have been presented for the elastic scattering form factors for the light $\mathrm{He}$ and $\mathrm{Li}$ isotopes, including those for the halo nuclei ${ }^{6} \mathrm{He}$ and ${ }^{11} \mathrm{Li}$, as well as the elastic scattering (longitudinal) form factor for ${ }^{8} \mathrm{~B}$. This is to encourage discussion of what measurements may be done at the SCRIT facility at RIKEN, as well as the proposed FAIR/ELISe experiment at GSI.

The effects on the neutron halo on the form factors is predicted to be negligible, as would be expected. However, the effect of the proton halo on the longitudinal form factor is predicted to be pronounced, as shown by the results for ${ }^{8} \mathrm{~B}$. This indicates that future experiments should place some emphasis on measurements of proton halos, as well as the possibility of progressing along an isotonic change, to see the effects of the changes the charge density as one increases the proton number. Work in the latter is underway with respect to some medium-mass isotonic chains as may be measured at the SCRIT facility.

If measurements of complementary intermediate-energy proton scattering data for the same nuclei are possible, together these would provide a map of the matter density of the nuclei. This would be a sensitive test for any structure model specified for exotic nuclei, whether they are halo or skin.

\section{References}

[1] S. Karataglidis and C. Bennhold, Probing proton halos through pion photoproduction, Phys. Rev. Lett., 80 (1998) 1614.

[2] S. Karataglidis, P. J. Dortmans, K. Amos, and C. Bennhold, Alternative evaluations of halos in nuclei, Phys. Rev. C, 61 (2000) 024319.

[3] T. Suda and M. Wakasugi, Structure studies of unstable nuclei by electron scattering, Prog. Part. Nucl. Phys. 55 (2005) 417.

[4] A. N. Antonov, et al., The electron-ion scattering experiment ELISe at the International Facility for Antiproton and Ion Research (FAIR) — a conceptual design study, Nucl. Instrum. Meth. A 637 (2011) 60.

[5] A. Lagoyannis, et al., Probing the ${ }^{6}$ He halo structure with elastic and inelastic proton scattering, Phys. Lett. B 518 (2001) 27.

[6] S. V. Stepansov, et al., 24.5A MeV ${ }^{6} \mathrm{He}+$ p elastic and inelastic scattering, Phys. Lett. B 542 (2002) 35.

[7] S. Karataglidis, K. Amos, B. A. Brown, and P. K. Deb, Discerning the neutron density distribution of ${ }^{208}$ Pb from nucleon elastic scattering, Phys. Rev. C 65 (2002) 044306.

[8] K. Amos, P. J. Dortmans, H. V. von Geramb, S. Karataglidis, and J. Raynal, Nucleon-nucleus scattering: a microscopic nonrelativistic approach, Adv. Nucl. Phys. 25 (2000) 275.

[9] T. deForest and J. D. Walecka, Electron scattering and Nuclear Structure, Adv. Phys. 15 (1966) 1.

[10] S. Karataglidis, P. Halse, and K. Amos, Transverse electric form factors for electron scattering and violation of current conservation in nuclear models, Phys. Rev. C 51 (1995) 2494. 
[11] Zaijin Wang, Zhongzhou Ren, and Ying Fan, Charge density distributions and charge form factors of the $N=82$ and $N=126$ isotonic nuclei, Phys. Rev. $C 73$ (2006) 014610.

[12] A. N. Antonov, et al., Charge and matter distributions and form factors of light, medium, and heavy neutron-rich nuclei, Phys. Rev. C 72 (2005) 044307.

[13] C. A. Bertulani, Probing nuclear skins and halos with elastic electron scattering, J. Phys. G 34 (2007) 315.

[14] M. Grasso, et al., Nuclear “bubble” structure in ${ }^{34}$ Si, Phys. Rev. C 79 (2009) 034318.

[15] S. Karataglidis and K. Amos, Electron scattering form factors from exotic nuclei, Phys. Lett. B650 (2007) 148.

[16] S. Karataglidis, B. A. Brown, K. Amos, and P. J. Dortmans, Shell model structures of low-lying excited states in ${ }^{6,7}$ Li, Phys. Rev. C 55 (1997) 2826.

[17] S. Karataglidis, private communication (2005).

[18] D. C. Zheng, B. R. Barrett, J. P. Vary, W. C. Haxton, and C.-L. Song, Large-basis shell model studies of light nuclei with a multi-valued G-matrix effective interaction, Phys. Rev. C 52 (1995) 2488.

[19] J. S. McCarthy, I. Sick, and R. R. Whitney, Electromagnetic structure of the helium isotopes, Phys. Rev. C 15 (1977) 1396.

[20] D. J. Millener, J. W. Olness, E. K. Warburton, and S. S. Hanna, Strong E1 transitions in ${ }^{9} B e,{ }^{11} B e$, and ${ }^{13}$ C, Phys. Rev. C 28 (1983) 497.

[21] L. R. Suelzle, M. R. Yearin, and Hall Crannell, Elastic electron scattering from ${ }^{6}$ Li and ${ }^{7}$ Li, Phys. Rev. 162 (1967) 992.

[22] J. Lichtenstadt, et al., High momentum transfer longitudinal and transverse form factors of the ${ }^{7} \mathrm{Li}$ ground state doublet, Phys. Lett. B219 (1989) 394.

[23] G. J. C. van Niftrik, L. Lapikás, H. de Vries, and G. Box, Magnetisation of the ${ }^{7}$ Li nucleus as obtained from electron scattering through $180^{\circ}$. The electric quadrupole moment of ${ }^{7}$ Li., Nucl. Phys. A174 (1971) 173. 